\title{
Application of Artificial Intelligence Recognition Technology in Digital Image Processing
}

\author{
Xi Zhang $(\mathbb{D}$ \\ Henan Medical College, Zhengzhou, 451191 Henan, China \\ Correspondence should be addressed to Xi Zhang; 2008050090@hamc.edu.cn
}

Received 3 November 2021; Accepted 3 January 2022; Published 27 January 2022

Academic Editor: Alireza Souri

Copyright (c) $2022 \mathrm{Xi}$ Zhang. This is an open access article distributed under the Creative Commons Attribution License, which permits unrestricted use, distribution, and reproduction in any medium, provided the original work is properly cited.

\begin{abstract}
Synthetic Artificial Intelligence technique is a science and technique derived and developed on the basis of calculator application technology. Image recognition is a special image processing step that plays an important role. Only after image recognition can it enter the stage of picture analysis and understanding. With the development of various computer technologies, images have gradually become and have become an important source of information for people. The use of calculator artificial intelligence is becoming increasingly widespread; therefore, understanding its application and related research is more conducive to pointing out the direction of research and learning for us. The goal of this paper is to discuss the emergence and development of synthetic intelligence identification technology and analyze the application bottlenecks of various types of synthetic intelligence identification technology, so as to increase our understanding of Synthetic Artificial Intelligence technique and provide reference for the research in related fields. This article simply introduces the technology of artificial intelligence type and its new development trend, and by combining concrete images of public facilities, the application of different computer artificial recognition methods of image recognition processing on the basis of the traditional method is improved, and through the corresponding simulation software of processing and identification methods for the analysis and comparison, the main application of two methods, the image processing recognition error rate is less than 0.5; improving computer artificial intelligence identification technique for the analysis of its application in image processing has certain help. The preprocessing process generally includes image digitization, grayscale, binarization, noise removal, and character segmentation. In terms of image recognition, algorithms mainly include statistical recognition, syntax recognition, and template matching. In recent years, with the development of neural networks and support vector machine technology, image recognition technology has a new and higher level of development.
\end{abstract}

\section{Introduction}

Artificial intelligence technology has been applied in more and more industries and fields, such as unmanned driving to bring changes to the transportation industry, the use of algorithm identification to help police arrest suspects, and intelligent robots to solve the problem of resource allocation in the medical industry. Artificial intelligence technology penetrates into all aspects of life and will bring great changes to the future society. For example, the evolution of domestic robots in China has made a breakthrough. Many families have started to use the sweeping robot as a necessary part of household cleaning. In the process of artificial intelligence design and optimization of household robots, the optimiza- tion of automatic recognition algorithm technology and visual interface design is always the core and key links. The so-called automatic recognition algorithm optimization refers to the realization that the new algorithm can be added to the algorithm framework of artificial intelligence timely through the system optimization of departments or all programs, so as to provide good online support for developers and help users become more familiar with the operation interface and the use of the framework. At present, in the frame design of domestic robots, there is obviously a problem of poor application effect in the object programming environment. For some wizard-style designs, there is often consistency and lack of differentiation, which leads to some modules becoming very cumbersome. This not only reduces 
the work efficiency of the robot, but also affects the user's experience.

Although there are many applications of image processing, the principles and methods used in them are the same, and there is no difference. Behind the high efficiency and high accuracy, there is still uncertainty in the artificial intelligence recognition technology. In the evolution of artificial intelligence technology, the inherent uncertainty of operating mechanisms makes the technology development similar to a "black box" state, so it is difficult for researchers to find the error and control the performance of the product. The uncertainty of exogenous variables mainly refers to the risk of technology development, among which the cost risk and schedule risk have the greatest impact. For artificial intelligence, even if its technology can be developed as expected, it still faces judgment on this technology. If it does not meet the moral and ethical needs of human beings, it will not be put into practice, leading to the failure of even successful development of the technology.

When subjected to a force that is parallel to the crosssection of the rod, close to each other, equal in magnitude, and opposite in direction, the deformation mainly characterized by the relative displacement of the cross-section of the rod is called shear deformation. This paper adopts the method of case study, based on the theories related to artificial intelligence and image recognition, through sorting out and analyzing the application status and problems of computer artificial intelligence technology in image processing, and puts forward the ideas and strategies of using artificial intelligence in the innovation of various industries.

\section{Related Work}

"Intelligent engineering," "computer study," and "deep training" are commonly referred to with different meanings in the field of high-level computational mathematics. Nawrocki et al. demythologized these expressions for radiation and established fundamental information about the topic. Not only that, they discuss the influence human performance may have on radiology in predicting the upcoming future. While ai is impossible to exchange radiologists anytime soon, Nawrocki et al. explore how technology could benefit the radiology field [1]. Mao et al. proposed instant sentiment identification method capture-based $2 \mathrm{~d}$ and $3 \mathrm{~d}$ features of faces' emotions by Kinect sensor. They combine the characters of Kinect's tracking unit (AUs) and feature point position (FPP). Tests on sensitive data systems and real-time videos show the best quality of this method [2]. Handwritten recognition is a method that allows a computer to automatically identify characters or scripts in the user language. Today, optical character recognition has become one of the most successful technological tools in process recognition and artificial intelligence. Here, handwritten English characters have been scanned and the image is typed into a computer, where it is recognized using a virtual network and converted to standard printed characters. To have an accurate measurement of partition and partition surprises and overcome the weaknesses of all other available OCR algorithms, Ananth et al. developed an approximate algo- rithm for each process [3]. Peng et al. believe that sheet metal forming failure may occur due to necking, fracture, or wrinkling. By using forming limit diagrams (FLD) as a powerful tool to prevent sheet metal failure during the forming process, it provides parameter control throughout the forming process. There are a variety of developed methods for predicting FLD that can estimate sheet metal forming strain limits. The evaluation of FLD estimates shows that there is a dependence between the effects of several factors including normal stress, shear stress, sheet thickness, mechanical properties, metallurgical properties, yield function, strain path, and bending and formability. In his research, the effect of bending was studied through two finite element models. In the first method, the effect of bending is studied by applying out-of-plane deformation by increasing the displacement of the punch. In the second method, the influence of bending is studied by changing the punch diameter $(25,50,70$, and $100 \mathrm{~mm})$ [4].

\section{Proposed Method}

\subsection{Computer Artificial Intelligence Recognition Technology}

\subsubsection{Types of Computer Artificial Intelligence Detection Technology}

(1) Voice Identification Technology. The voice recognition technology is mainly based on the characteristics of the voice of the different age groups, respectively, voice recognition, the sound of the information, and the information of the voice recognition system data of a unified matching, so as to better realize the voice recognition and identification of the object, can the voice recognition technology are mainly on the basis of person more accurately identifying the crowd.

(2) Fingerprint Identification Technology. This fingerprint identification technology mainly uses the identification of fingerprints of different colors, because the color fingerprint of every ordinary person is a unique identification symbol of each person, and the technology of this color fingerprint identification can identify a person's true identity with high reliability and accuracy [5].

(3) Face Recognition Technology. Face recognition combines people's main facial features and pupil conditions to identify objects. The technique is also relatively accurate. In recent years, it is also in the stage of rapid development and has been widely applied [6].

(4) Smart Card Recognition Technology. Smart card recognition technology stores and calculates data through an integrated circuit board and collects, analyzes, and organizes different data. This recognition technology mainly relies on network information data. At present, smart card recognition technology is mainly used for vehicle recognition.

(5) Barcode Recognition Technology. Barcode identification includes one-dimensional and QR tag techniques. QR tag technique is an extension and development of one- 
dimensional code technology, with higher accuracy and more powerful functions, such as more powerful information capacity and error correction function [7].

(6) Rfid Technology. Radiofrequency technology mainly uses wireless electromagnetic waves to realize target recognition. The working principle of this technology is in corresponding tags; electromagnetic fields are used to transmit radio signals for data tracking and automatic identification [8].

\subsubsection{Application of Artificial Smart Identification Skills of PC}

(1) Application in the Field of Voice Speaker Detection. Nowadays, voice recognition technology includes voice search systems. The technology related to voice control can indeed bring more convenience to people, but the use of voice remote technology still has the problem of instability. In the course of advances in engineering, the existing problems cannot be ignored, which still need the research and efforts of the technicians in the current field. Not only the corresponding vocabulary should be enriched but also the fuzzy sound recognition ability should be strengthened to improve the speech recognition system.

(2) Application in terms of Image Recognition. Nowadays, the use of artificial intelligence image recognition technology in the field of image recognition technology is very limited, because image recognition technology is more difficult than other artificial intelligence recognition technologies. These technologies include, for example, the widely used vehicle license plate recognition system; Animal electrocardiogram recognition technology extensive range of materials for industrial purposes, farm and medical health; Seed and plant identification techniques, agriculture and information technology; mobile face recognition technology and mobile fingerprint recognition technology are widely used in the field of transportation and public safety management. But its current technology development status and trends, image recognition in the technology development, a primary stage, factors such as color and image contrast great extent hindered the further development of the recognition technology, technical personnel at the time of learning and using the image recognition technology but also on the transformation of a series of information, such as dimension reduction operation, this greatly reduces the degree of its efficiency, does not conform to the trend of the development of the era, is also difficult to well meet the demand of people's recognition of this age $[9,10]$.

(3) Application in the Field of Robotics. The BP network can learn and store a large number of input-output pattern mapping relationships without revealing the mathematical equations describing this mapping relationship in advance. Its learning rule is to use the steepest descent method to continuously adjust the weights and thresholds of the network through backpropagation to minimize the sum of squared errors of the network. The topological structure of the BP neural network model includes the input layer, hidden layer, and output layer.

Due to the lack of accurate understanding and exploration of human beings, robots are sometimes too slow and inefficient in the process of learning and working and fail to fully exploit the significance and strengths of synthetic intellectual property. So, in the artificial intelligence exploration of technical personnel late even more pay attention to identify accurate perception and functions of the artificial intelligence technology and simulation for the accurate understanding of thinking, the key is simulated by thinking, better will be a combination of human and other artificial intelligence technology, map the process of the consciousness and behavior of the robot to artificial intelligence, computer program, believed this is not only beneficial to help the artificial intelligence technology to get the breakthrough, also to help people to better understand and enjoy the use of artificial intelligence, computer, so as to better improve the quality of work and life [11,12].

\subsection{Image Recognition}

3.2.1. Composition of Image Recognition Technology. The image pattern is the definition and description of the image object to be recognized. The image pattern class is the collection of sample objects with some common characteristics of geometric, texture, and mathematical description. Image pattern recognition is the procedure of manipulation and evaluation of all forms of material data and graphic information on behalf of things or political entities in order to classify, evaluate, and illustrate images. An example of a study on graphic pattern discrimination is a process of automatic recognition and classification of the geometric target, regional texture, and mathematical description target in object image by the comprehensive application of image processing, feature definition and transformation, classification method, digital calculation, and other technologies.

General image pattern recognition systems are mainly composed of parts, including capture of picture images, picture presorting, extraction of image character, design of categorizer, and decision-making [13].

Common methods of image recognition are shown in Table 1.

\subsubsection{Synthesis of Intelligent Artificial Identification Methods in Image Vision Assessment Process}

(1) Smallest Range Categorizer. The smallest range categorizer is a facile pattern classifier, which estimates the statistical parameters of various patterns based on the sampling of the patterns and is completely determined by the means and variances of various types $[14,15]$. Assume that every mode level is characterized by a parameter of the mean magnitude vector:

$$
m_{j}=\frac{1}{N_{j}} \sum_{x \in S_{j}} x, \quad j=1,2, \cdots, M
$$


TABLE 1: Common methods of image recognition.

\begin{tabular}{lccc}
\hline Methods & Surface features & Identify ways & Typical criterion \\
\hline Template matching & Samples, pixels, curves & Correlation coefficient, distance measurement & Classification error \\
Statistical recognition & Characteristics & Classifier & Classification error \\
Structure identification & Tectonic language & Rules and grammar & Acceptable error \\
The neural network & Samples, pixels, features & Network function & Minimum root mean square error \\
\hline
\end{tabular}

where $N_{j}$ Indicates the maximum amount of schemas in the category $S_{j}$. The methodology for sorting an unlimited set of modality vectors is to designate the module to its corresponding group $[16,17]$. Using Euclidean distance to determine the degree of proximity, the problem is transformed into the measurement of distance:

$$
D_{j}(x)=\left\|x-m_{j}\right\|, \quad j=1,2, \cdots, M .
$$

Since the smallest distance represents the best match, if $D_{j}(x)$ is the smallest distance, then $x$ is assigned to class $S_{j}$. Equivalent to calculation $[18,19]$ :

$$
d_{j}(x)=x^{T} m_{j}-\frac{1}{2} m_{j}^{T} S_{j}, \quad j=0,1, \cdots, m .
$$

And assign $x$ to class $S_{j}$ when $D_{j}(x)$ gives the maximum value. For a minimum classifier, the decision boundary between class $S_{j}$ and class $S_{j}$ is

$t_{m n}(a)=t_{i}(a)-t_{j}(a)=a^{T}\left(n-n_{j}\right)-\frac{1}{2}\left(a_{i}-a_{j}\right)^{T}\left(a_{i}-a_{j}\right)=1$.

(2) Bayes Classifier. Statistical dispersion of Known and these types in the feature language of $d d M$ class items, i.e. with known typology $w_{i}=1,2, \cdots, M$ priori probability and class condition probability density $[20,21]$, for the specimen to be tested, the probabilities that the probability that the prototype belonging to each of the other sorts can be derived from the Bayesian formula, the experimental likelihood that the identification object exists can be generated to test the most probable $X$ attributes of the class to which the prototype belongs, the most likelihood that $X$ is of the type [22]. If class $M w_{i}$ is reciprocal and perfect, the Bayesian formula is formulated as follows:

$$
q\left(w_{i} \mid m\right)=\frac{P\left(X \mid w_{i}\right) P\left(w_{i}\right)}{\sum_{j=1}^{M} P\left(X \mid w_{j}\right) P\left(w_{j}\right)} .
$$

In engineering application problems, the data typically complies with a regular classification, which is justified and generalized in its physics, and the distribution is straightforward to manipulate math-wise. $N\left(u, \sigma^{2}\right)$ only consists of two parametrizations, which can be acquired by the expectations and covariances in the function of a large population of samples estimated to establish the density of dependent odds
[23]:

$$
P(x)=\frac{1}{\sqrt{2 \pi \sigma}} \exp \left[-\frac{1}{2}\left(\frac{u-1}{\sigma}\right)^{2}\right]=N\left(a, u^{2}\right),
$$

where $u$ is the mean or mathematical expectation:

$$
u=E(x)=\int_{-\infty}^{\infty} x P(x) d x
$$

The normative odds ratio diversity feature for covariates is as follows:

$$
P(x)=\frac{1}{(2 \pi)^{n / 2}|S|^{1 / 2}} \min \left[-\frac{1}{2}(Y-\bar{x})^{T} S^{-1}(Y-\bar{x})\right] .
$$

Denoted by probability diversity of variables in multidimensional orthonormal odds density features:

$$
P\left(x \mid w_{i}\right)=-\frac{1}{2}\left(Q-\overline{X_{i}}\right)^{T} S_{i}^{-1}\left(Q-\overline{X_{i}}\right)-\frac{n}{2} \ln 2 \pi-\frac{1}{2} \operatorname{lm}\left|S_{i}\right| .
$$

The posterior probability can be calculated. It represents the likelihood that the target panel falls into various categories when it presents $X$ characteristic state. The characteristic $X$ of $A$ sample may be represented in whole or in part in class $M$, while the probability of its representation in class $w_{i}=1,2, \cdots, M$ is expressed in terms of $P\left(X \mid w_{i}\right)$. The posterior probability expressed by Bayes formula:

$$
F\left(v_{i} \mid y\right)=\frac{P\left(y \mid v_{i}\right) P\left(v_{i}\right)}{\sum_{j=1}^{M} P\left(y \mid v_{j}\right) P\left(v_{j}\right)} .
$$

It represents $A$ conditional probability, representing the approximate sample belonging to the class $A$ in the case of $y$ feature, and it can be estimated in turn the category of the sample to be tested that has $A$ certain feature $y$. The mathematical formula is used to express the second kind of problem:

$$
\begin{array}{ll}
P\left(v_{i} \mid y\right)>P\left(v_{2} y\right), & a \in v, \\
P\left(v_{i} \mid y\right)<P\left(v_{2} \mid y\right), & a \in v_{2} .
\end{array}
$$

The structure of the Bayesian classifier for the two types of problems is shown in Figure 1.

The classifier of the nervous system module distribution is shown in Figure 2; the geometry of the neuron network 


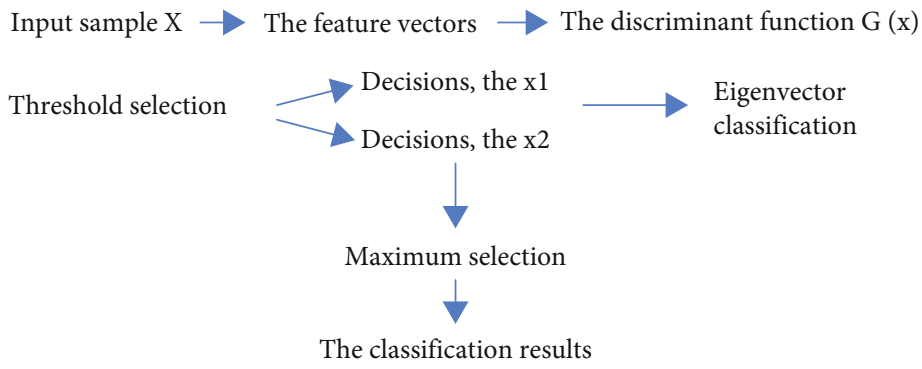

Figure 1: Structure of the Bayes classifier for multiclass problems.

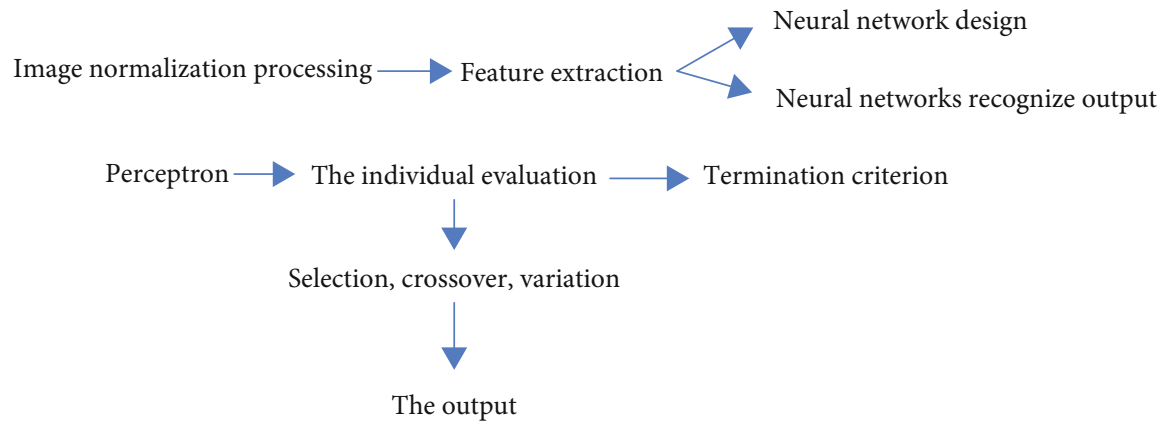

FIGURE 2: Components of the recognition system module based on neural network.

TABLE 2: Correspondence between biological evolution and genetic algorithm.

\begin{tabular}{|c|c|}
\hline $\begin{array}{l}\text { Concepts in biological } \\
\text { evolution }\end{array}$ & The role of genetic algorithms \\
\hline The environment & To adapt to the function \\
\hline Adaptive & Adaptive value function \\
\hline The survival of the fittest & The solution with the greatest value of the adaptive function has the greatest probability of being retained \\
\hline Individual & One solution to the problem \\
\hline Chromosome & Solution of the coding \\
\hline Gene & Coded element \\
\hline Group & A selected set of solutions \\
\hline
\end{tabular}

discriminant function classifier does not need to rely on the conditional probability density of the traditional statistical scientific knowledge; a classifier model by some kind of conditional probability transformation maps the dividing line between the feature vectors in a pattern; the pattern of spatial feature vector can be understood as belonging to a point, in the feature space, which belongs to a feature point set in the feature space to a certain extent which is always associated with another type of feature point set or separation; each model in mathematics and geometry relationship between the classes is to determine the separable. It can be understood that a dividing line can be found through the linear classification method of mathematics and geometry, and the class belonging to the feature space can be decomposed into a subfeature space corresponding to different categories. Another result of geometric linear classification is the ability to provide a definite dividing line equation, called a discriminant function, for an object. According to the property and modality of linear discriminant filters, sorting can be classi- fied into linear discipline segmentation functions and nonlinear differentiation segmentation factors. A geometric linear criterion function categorizer is widely used in desktop computers and for pattern recognition of images for its ease of interpretation and integration in a traditional design.

(3) Genetic Algorithm. The most critical parts of the genetic algorithm are selection, crossover, and variation. Its performance is mainly affected by these three genetic operations, and its corresponding relationship is shown in Table 2. Crossover is the selection of genes from parental chromosomes to create an intermediate individual by one-point traverse, bipoint intersection, $n$-point intersession, uniform intersection, and operator's intersection. The mutation occurs after the crossing. This is to avoid that all in the total number of solutions fall into the local optimal problem for the one being worked on. Mutation is a stochastic change of intermediate individuals. It is mainly to duplicate the best centromere, and there are many approaches, such as rotary selectivity, local 


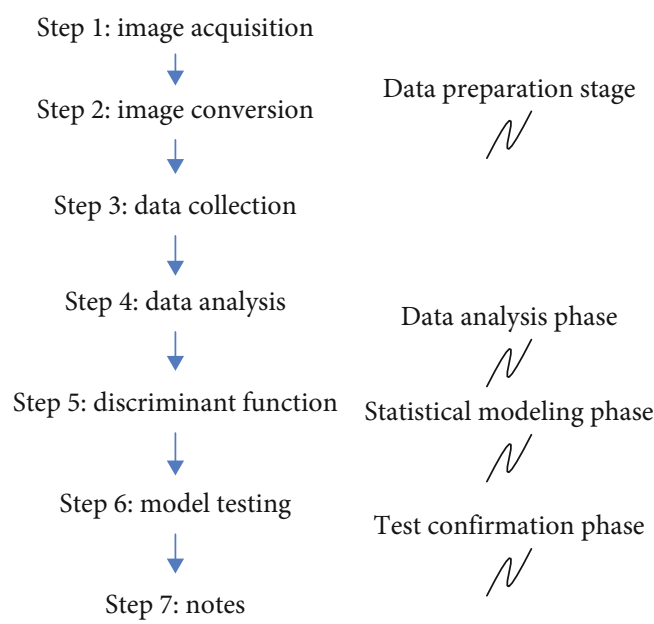

FIgURe 3: Experimental design steps.

kinematic selection, championship kinematic conversion, transitional selection, and steady-state choice. Taking roulette selection as an example, imagine placing all the chromosomes in a population on a roulette wheel, where each individual's chromosomes have their place in the fitness function, and parental selection in terms of fitness; the better the chromosome, the better the chance of being selected. That is, when a pinball is thrown to select chromosomes, chromosomes with greater fitness will be selected multiple times. Since genetic algorithms cannot directly deal with the parameters of the problem space, the problem to be solved must be expressed as a chromosome or individual in the genetic space through coding. This conversion operation is called encoding, and it can also be called (representation of the problem). The following three criteria are often used to evaluate coding strategies: (a) Completeness: all points (candidate solutions) in the problem space can be represented as points (chromosomes) in the GA space. (b) Soundness: the chromosomes in the GA space can correspond to all candidate solutions in the problem space. (c) Nonredundancy: chromosomes correspond to candidate solutions one-to-one.

\section{Experiments}

4.1. Experimental Background. In the contemporary world, the safety of utilities plays a more critical role in the city context, and its inspection skills are growing in salience in the system, and it has become an instrument to control the quality of products and guarantee the safe facility running. Its important function depends on the correctness of test method selection and the reliability of test results. In the detection of most public infrastructure such as roads and bridges, if the detection personnel only rely on their own vision to observe and expect to get some useful information, a lot of subjective factors will be introduced in the detection process due to human participation, which will eventually make the detection quality of facilities uncertain. And this method workload is big, has poor reliability, and rely on repair personnel's experience and sense of responsibility, work efficiency is low, long repeat job easily causes visual fatigue, and in many cases due to potential risk in the work environment, such as the traffic on the highway,

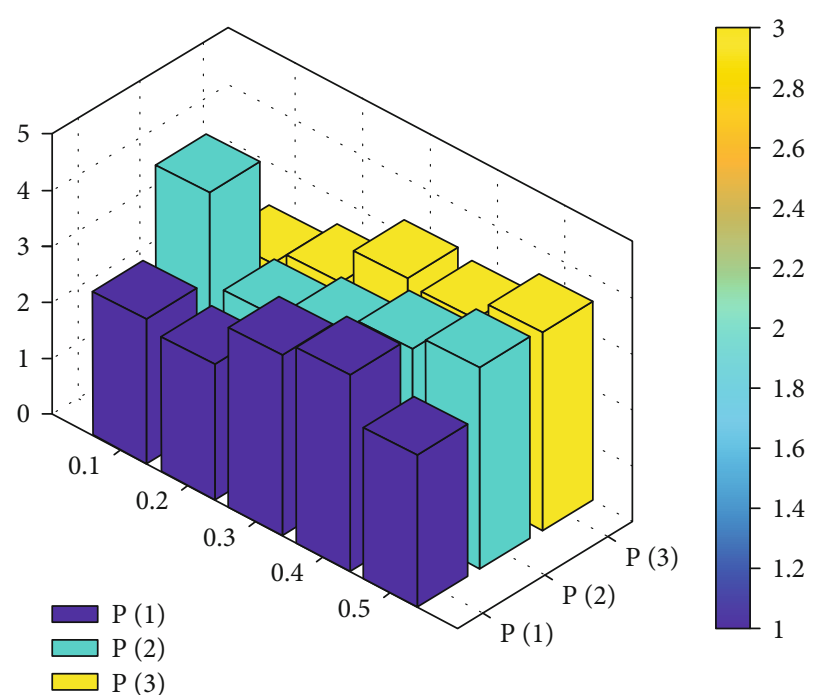

FIgURE 4: Sample feature distribution after three times of sample $t$ input.

the work high above is hazardous to humans. Therefore, in order to minimize the adverse impact of human factors in the testing process, the development of automated testing technology is a vital way to improve the reliability of testing results. According to the introduction of computers, automatic detection and identification can improve the reliability and security of facility detection, save manpower and labor, and improve social benefits.

4.2. Experimental Design. Automatically detect and identify the facilities by computer, which can improve the recognition efficiency and guarantee the safety of personnel. It has implications in the field. Four phases of application engineering are undertaken: data generation, data analyzing, counting, simulation, and confirming (see Figure 3).

4.2.1. Take Pictures of Steel Bridge Coating Surface. The selected steel bridges were coated with a layer of blue paint, and two sets of pictures were taken with digital cameras: a set of intact samples and a set of defective samples, each set of 10. In the flawed pictures, use the pictures with slight rust as much as possible to increase the efficiency of the model, so that the model can be maintained in advance at the initial stage of the defect.

4.2.2. Image Conversion. By converting the color image to the RGB chromaticity space, you can obtain the pixel value $x_{i j}$ of the image on the three color channels of RGB. I represents the color channel $=$ red, green, and blue, and $j$ represents the picture $=1,2, \cdots, n$.

4.2.3. Extract Statistical Data. Three statistical values were extracted from each color channel to show the characteristics of the image, namely, difference (DIFF) division, mean, and standard deviation (STD).

The nine statistical features obtained in the previous step would make the dimension too high if used directly in modeling and would be further reduced based on the analysis. The purpose of data analysis and selection is to select effective 


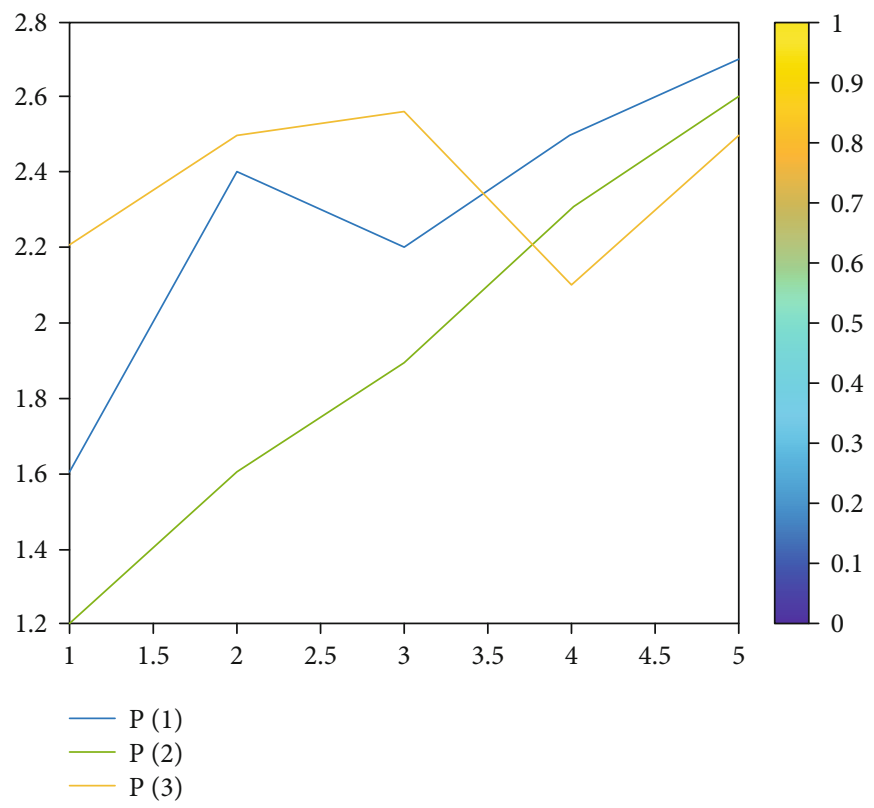

FIgURE 5: Error curves under different samples.

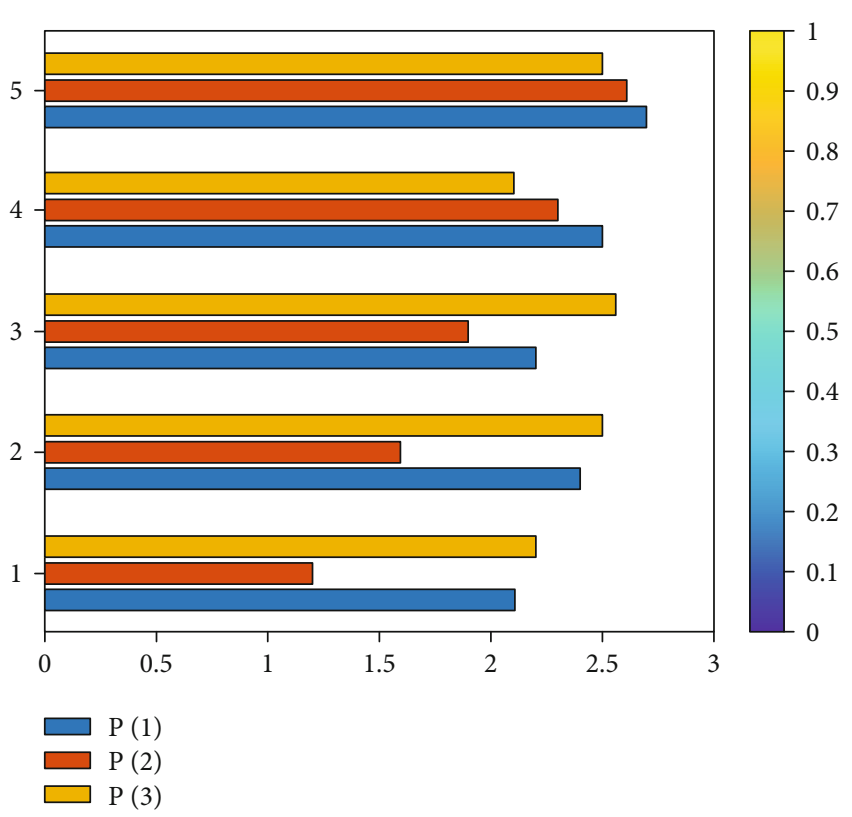

FIGURE 6: Variation diagram of network training target error under BP algorithm.

feature variables from individual variables. The following analysis method is used to quantitatively test the mean difference between different groups. The basis is that the greater the mean difference of the same feature variable in different groups, the greater the contribution of the variable to the discriminant function, and the more effective the feature vector.

\section{Discussion}

5.1. Computer Artificial Intelligence Recognition Technology Based on Perceptron. The recognition algorithm of Bayesian decision theory is a relatively basic algorithm in the statisti- cal pattern recognition algorithm. Since the process of obtaining the linear discriminant function in the algorithm is equivalent to the learning process of the perceptron, the perceptron neural network can also be introduced to realize this kind of image recognition problem.

For 20 samples, we can know the matrix of the perceptron's input vector $p$. Since the problem has been classified into a simple two-category recognition problem, the corresponding target value of the target vector can be selected as 1 and 0 to represent the classification. Let us assume that 1 represents the class of images that are intact, and 0 represents the class of images that are defective. According to the input vector $t$, a corresponding target vector can be obtained and a single-layer perceptron neuron can be selected, as shown in Figure 4 in the sample feature distribution map after the input vector $t$.

Notice a phenomenon in the perceptron algorithm, that is in different initial conditions $w, b$, the training result is different, but after training, the network can complete the classification task, only steps needed for the training, and finally the result is likely to be different, namely the classification problem of only one solution, on the premise of the four steps to achieve results, and the error change as shown in Figure 5.

\subsection{Analysis of Computer Artificial Intelligence Recognition} Technology Based on BP Neural Network. The basic idea of the BP algorithm is that the learning process consists of two processes: the forward propagation of the signal and the backpropagation of the error. In the forward propagation, the input samples are passed in from the input layer, processed by each hidden layer by layer and then passed to the output layer. If the actual output of the output layer is inconsistent with the expected output (teacher signal), it will turn to the error backpropagation stage. Error backpropagation is to pass the output error back to the input layer by 


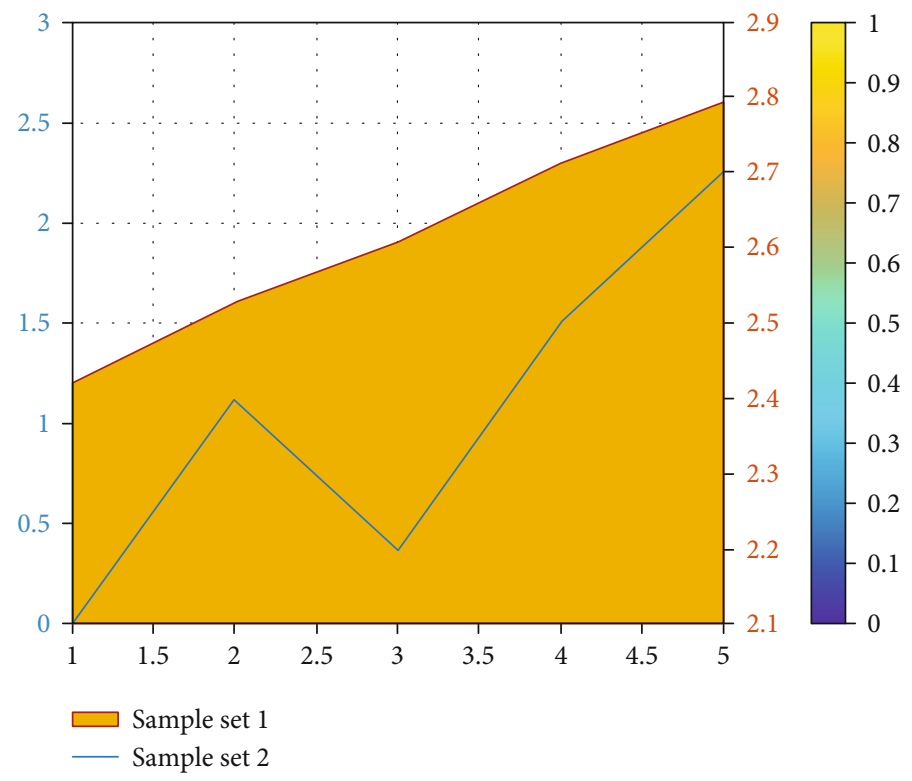

Figure 7: 20 network output changes of 20 samples.

TABle 3: The degree of closeness between the image to be tested and the known sample.

\begin{tabular}{lccc}
\hline Sample & $x$ & Sample & $x$ \\
\hline 1 & 0.2356 & 8 & 0.2236 \\
2 & 0.3991 & 9 & 0.3255 \\
3 & 0.4168 & 10 & 0.3412 \\
4 & 0.2881 & 11 & 0.3259 \\
5 & 0.2447 & 12 & 0.2529 \\
6 & 0.3052 & 13 & 0.3510 \\
7 & 0.4033 & 14 & 0.3510 \\
\hline
\end{tabular}

layer through the hidden layer in some form and apportion the error to all the units of each layer, so as to obtain the error signal of each layer unit, and this error signal is used as a correction for each unit as the basis of the weight. The process of adjusting the weights of each layer of signal forward propagation and error backpropagation is carried out repeatedly. The process of constant weight adjustment is the learning and training process of the network. This process continues until the error of the network output is reduced to an acceptable level or until the preset number of learning times.

For solving two kinds of problems, the characteristic variables are linearly combined into a standard discriminant function. Since the eigenvalues of the training data can maximize the separation of the two classes, the coefficients of the discriminant function are optimal. Linear discriminant analysis does not change the location of the original data, it simply attempts to provide a higher degree of separation and to carve out a decision area for a given class, so this approach can better help understand the distribution of the characteristic data. The feature vectors in the input layer refer to the feature extraction in the previous algorithm, and three fea- tures extracted from the color image are used. In Kolmogorov's theorem, the number of hidden layer neurons is selected as 7; for the output layer, you only need to divide the image into two categories, so you only need to use one neuron as the output.

Parameters of the BP network are set as follows.

The maximum number of training steps in the network is net.trainparam.epochs $=1000$; the training target error is net. TrainParam.Goal $=0.001$.

Figure 6 is the error variation diagram of the network training target. Figure 7 is the network training output corresponding to the 20 samples collected previously. It is obvious that the training samples are two sample set elements: the first 10 samples are the elements of sample set 1 , and the last 10 samples are the elements of sample set 2 . It can be regarded as a whole; that is, there is a sample element in the total sample set, the fuzzy feature vectors are extracted, and then, the closeness between the image to be tested and the known sample image is calculated by using the fuzzy method, and the classification is conducted according to the principle of proximity selection.

Due to image is a known samples, each sample corresponds to a matrix, such known samples of the fuzzy set is expressed as a matrix, the corresponding eigenvector extracted under test images, according to the degree calculation sample under test and the known sample set close to the extent, maximize is belong to category of the sample under test, the degree of the pattern and the known sample under test as shown in Table 3.

According to the above process, the 20 images in the known sample set were taken as the test samples for classification and recognition, and input was selected randomly, the closeness degree was calculated, the maximum value and the corresponding known sample serial number were found, and the correct category could be obtained. The above algorithms have something in common; they all use the same sample eigenvectors. The feature vector is extracted 
from color image RGB three channel information, but because of its high correlation between RGB, and then use the Wilks' lambda criteria, to gradually reduce the correlation characteristic vector, dimension reduction, for each color images can use three feature vector to represent the main part of information.

\section{Conclusions}

The biggest difference between a digital image and an analog image is that it can be stored on a computer and can be coded and modified using image editing software. How to effectively process images has also become a research hotspot and focus of computer technology. Although a lot of artificial intelligence recognition technology has been applied, at the same time, there are still some shortcomings at the technical level. For example, the voice recognition system, which is mainly applied to putonghua due to the restrictive types of identifiable languages, will not be able to accurately identify regional accents or nonstandard pronunciation, and the application effect will be significantly reduced. At the same time, for face recognition technology widely used, the database cannot cover all facial expression features; limited by data, the recognition effect cannot be guaranteed. However, in the case of increasing age, facial features will also change to some extent, which is also one of the factors reducing the recognition effect. Face recognition technology mainly relies on visual features, which cannot exclude the factor of face similarity, and the interference of other scene factors will increase the recognition error rate. It can be seen from this that on the basis of summarizing previous experience, it is necessary to conduct more in-depth research on the artificial intelligence recognition technology, so as to further improve the comprehensive level of technology while eliminating the existing defects.

This paper briefly introduces artificial intelligence recognition technology and image processing technology and focuses on the application of artificial intelligence recognition technology in image processing. Regular safety inspection of public facilities is an important means to control product quality and ensure the safe operation of facilities. In order to minimize the adverse impact of human factors on testing and improve the reliability of testing results, developing automatic testing technology is an effective way to solve the problem. In the surface quality inspection of highways, bridges, and other facilities, image recognition technology is used to detect the target image automatically. In this paper, according to the specific color images collected in the detection, different identification methods are used to realize the detection, so as to improve the reliability of the detection results and shorten the identification time and at the same time deepen the understanding of the application of image recognition technology.

The main content of this article is the research of image filtering and image recognition algorithms. Image recognition is a type of pattern recognition. Image recognition technology has been successfully used in fields such as optical information processing, medical instrument sample inspection and analysis, automated instruments, industrial inspection, license plate recognition, and unmanned driving at home and abroad. As an important aspect of artificial intelligence, image recognition has always been widely concerned and highly valued by people. Image recognition includes two steps: image preprocessing and image recognition. Image filtering is a crucial step in image preprocessing. The end of this article through to the public facilities to recognition of color images collected in detection, using the Matlab simulation, to change the traditional method, and using method of bayes, sensors, the BP neural network and the fuzzy recognition method of image recognition, and for the various intelligent method of intelligent identifying new FaZhanDian namely combination gives the research difficulty and the current development of hot spots, more fully expounds the application of intelligent technology in image processing. In the algorithm for removing Gaussian noise, the edge of the image is not effectively protected, which makes the edge have a certain degree of distortion. Although the algorithm proposed in this study shows better results than the traditional algorithm in the simulation experiment, the algorithm itself does exist many shortcomings that need to be improved in future work.

\section{Data Availability}

This article does not cover data research. No data were used to support this study.

\section{Conflicts of Interest}

The author declares no potential conflicts of interest with respect to the research, authorship, and/or publication of this article.

\section{References}

[1] T. Nawrocki, P. D. Maldjian, S. E. Slasky, and S. G. Contractor, "Artificial intelligence and radiology: have rumors of the radiologist's demise been greatly exaggerated?," Academic Radiology, vol. 25, no. 8, pp. 967-972, 2018.

[2] Q. Mao, X. Pan, Y. Zhan, and X. J. Shen, "Using Kinect for real-time emotion recognition via facial expressions," Frontiers of Information Technology \& Electronic Engineering, vol. 16, no. 4, pp. 272-282, 2015.

[3] J. P. Ananth, G. Raghuraman, G. L. I. Cyril, and M. S. Aldo, "Enhancement of segmentation and zoning to improve the accuracy of handwritten character recognition," Journal of Computational and Theoretical Nanoscience, vol. 12, no. 12, pp. 5891-5894, 2015.

[4] D. Peng, S. Chen, R. Darabi, A. Ghabussi, and M. Habibi, "Prediction of the bending and out-of-plane loading effects on formability response of the steel sheets," Archives of Civil and Mechanical Engineering, vol. 21, no. 2, pp. 1-13, 2021.

[5] W. Sureshkumar and R. Rama, "Chomsky hierarchy control on isotonic array P systems," International Journal of Pattern Recognition and Artificial Intelligence, vol. 30, no. 2, pp. 1650004-1650249, 2016.

[6] L. Zhou, G. Yang, Y. Yin, L. Yang, and K. Wang, "Finger vein recognition based on stable and discriminative superpixels," International Journal of Pattern Recognition \& Artificial Intelligence, vol. 30, no. 6, pp. 1650015-1650079, 2016.

[7] Y. Zhang-Jing, H. Pu, and Z. Fan-Long, "Center-based line neighborhood discriminant embedding algorithm and its 
application to face recognition," Pattern Recognition \& Artificial Intelligence, vol. 21, no. 3, p. 65, 2015.

[8] D. Verma and S. Dubey, "Fuzzy brain storm optimization and adaptive thresholding for multimodal vein-based recognition system," International Journal of Pattern Recognition \& Artificial Intelligence, vol. 13, no. 5, pp. 49-61, 2016.

[9] F. Tian, Y. Gao, Z. Fang, and J. Gu, "Automatic coronary artery segmentation algorithm based on deep learning and digital image processing," Applied Intelligence, vol. 51, no. 12, pp. 8881-8895, 2021.

[10] E. Giacomin, T. Greenberg-Toledo, S. Kvatinsky, and P.E. Gaillardon, "A robust digital RRAM-based convolutional block for low-power image processing and learning applications," IEEE Transactions on Circuits and Systems I: Regular Papers, vol. 66, no. 2, pp. 643-654, 2019.

[11] Z. Che and X. Zhuang, "Digital affine shear filter banks with 2layer structure and their applications in image processing," IEEE Transactions on Image Processing, vol. 27, no. 8, pp. 3931-3941, 2018.

[12] L. Zhang, L. Zhang, and L. Zhang, "Application research of digital media image processing technology based on wavelet transform," EURASIP Journal on Image and Video Processing, vol. 2018, no. 1, 2018.

[13] R. Mouhcine, A. Mustapha, and M. Zouhir, "Recognition of cursive Arabic handwritten text using embedded training based on HMMs," Journal of Electrical Systems \& Information Technology, vol. 32, no. 1, p. 455, 2017.

[14] K. Tripathi and N. B. G. C. Nandi, "Continuous Indian sign language gesture recognition and sentence formation," Procedia Computer Science, vol. 54, no. 2, pp. 523-531, 2015.

[15] R. Malik, "AI augmentation in the field of digital image processing," International Journal of Computer Applications in Technology, vol. 65, no. 3, pp. 235-244, 2021.

[16] F. Boutekkouk, "Digital color image processing using intuitionistic fuzzy hypergraphs," International Journal of Computer Vision and Image Processing (IJCVIP), vol. 11, no. 3, pp. 2140, 2021.

[17] S. S. Rautaray and A. Agrawal, "Vision based hand gesture recognition for human computer interaction: a survey," Artificial Intelligence Review, vol. 43, no. 1, pp. 1-54, 2015.

[18] R. Herrera-Pereda, A. Taboada Crispi, D. Babin, W. Philips, and M. Holsbach Costa, "A review on digital image processing techniques for in-vivo confocal images of the cornea," Medical Image Analysis, vol. 73, article 102188, 2021.

[19] İ. İlhan, "A cross-platform test tool for digital image processing," Multimedia Tools and Applications, vol. 80, no. 8, pp. 12249-12273, 2021.

[20] L. Ma, "Research on distance education image correction based on digital image processing technology," EURASIP Journal on Image and Video Processing, vol. 2019, no. 1, 2019.

[21] P. Shamsolmoali, M. E. Celebi, and R. Wang, "Deep learning approaches for real-time image super-resolution," Neural Computing and Applications, vol. 32, pp. 14519-14520, 2020.

[22] M. A. Borkin, Z. Bylinskii, and N. W. Kim, "Beyond memorability: visualization recognition and recall," IEEE Transactions on Visualization \& Computer Graphics, vol. 22, no. 1, pp. 519528, 2016.

[23] Z. Yang, L. Yang, W. Huang, L. Sun, and J. Long, "Enhanced deep discrete hashing with semantic-visual similarity for image retrieval," Information Processing and Management, vol. 58, no. 5, article 102648, 2021. 